TRANSACTIONS OF THE

AMERICAN MATHEMATICAL SOCIETY

Volume 362, Number 3, March 2010, Pages 1189-1210

S 0002-9947(09)04769-2

Article electronically published on September 23, 2009

\title{
THE LOWER BOUND OF THE $w$-INDICES OF SURFACE LINKS VIA QUANDLE COCYCLE INVARIANTS
}

\author{
MASAHIDE IWAKIRI
}

\begin{abstract}
The $w$-index of a surface link $F$ is the minimal number of the triple points of surface braids representing $F$. In this paper, for a given 3-cocycle, we consider the minimal number of the $w$-indices of surface links whose quandle cocycle invariants associated with $f$ are non-trivial, and denote it $\omega(f)$. In particular, we show that $\omega\left(\theta_{3}\right)=6$ and $\omega\left(\theta_{p}\right) \geq 7$, where $\theta_{n}$ is Mochizuki's 3 -cocycle of the dihedral quandle of order $n$ and $p$ is an odd prime integer $\neq 3$. As a consequence, for a given non-negative integer $g$, there are surface knots with genus $g$ with the $w$-index 6 .
\end{abstract}

\section{INTRODUCTION}

A surface link is a closed oriented surface embedded in Euclidean 4-space $\mathbf{R}^{4}$ locally flatly. Two surface links $F$ and $F^{\prime}$ are equivalent if there is an orientationpreserving homeomorphism $h: \mathbf{R}^{4} \longrightarrow \mathbf{R}^{4}$ such that $h(F)=F^{\prime}$. A closed surface braid of degree $m$ is a closed oriented surface embedded in $D_{1} \times U_{0}$ locally flatly such that the restriction map $\left.\pi\right|_{S}$ of the projection map $\pi: D_{1} \times U_{0} \longrightarrow U_{0}$ is a simple $m$-fold branched covering map where $D_{1}$ is a 2-disk and $U_{0}$ is a 2 -sphere. Two surface braids $S$ and $S^{\prime}$ with the same degree are equivalent if they are ambient isotopic by a fiber-preserving isotopy $\left\{h_{u}\right\}_{0 \leq u \leq 1}$ of $D_{1} \times U_{0}$, as a $D_{1}$-bundle over $U_{0}$. Alexander's theorem in dimension four, i.e., any surface link is equivalent to a closed surface braid of a certain degree as surface links, was announced in [18] and proved in [10.

By the $w$-index of a closed surface braid $S$, we mean the minimal number of triple points of $S^{\prime}$ such that $S^{\prime}$ is equivalent to $S$. The minimal number of the $w$-indices of closed surface braids equivalent to a surface link $F$ is called the $w$-index of $F$, which is denoted by $w(F)$. S. Kamada 8 proved that a surface link $F$ is ribbon if and only if $w(F)=0$. I. Hasegawa [6] proved that the $w$-index of a non-ribbon surface link is at least four and the $w$-index of a non-ribbon spherical link is at least six. M. Ochiai, T. Nagase and A. Shima [15] proved that there is no surface link whose $w$-index is five.

J. S. Carter, D. Jelsovsky, S. Kamada, L. Langford and M. Saito 2] defined the quandle cocycle invariant $\Phi_{f}(F)$ of a surface link $F$ associated with an $A$-valued quandle 3-cocycle $f$, where $A$ is an Abelian group. See 2 for the original definition of $\Phi_{f}(F)$. The quandle cocycle invariant $\Phi_{f}(F)$ is considered as a multi-set of $A$

Received by the editors June 4, 2007.

2000 Mathematics Subject Classification. Primary 57Q45.

This paper was supported by JSPS Research Fellowships for Young Scientists and the 21 COE program "Constitution of wide-angle mathematical basis focused on knots".

(C)2009 American Mathematical Society Reverts to public domain 28 years from publication 
as in [4. By the definition of $\Phi_{f}(F)$, we see that if the triple point number of $F$ is zero, then every element in $\Phi_{f}(F)$ is zero. Here, we have a natural question, i.e., for a given 3-cocycle $f$, how many is the minimal number $\tau(f)$ of the triple point number of surface links whose quandle cocycle invariants associated with $f$ include a non-zero element? The answer to this question is known for a few 3-cocycles as follows: S. Satoh and A. Shima [16, 17] proved that $\tau\left(\theta_{3}\right)=4$ for Mochizuki's 3 -cocycle $\theta_{p}$ of the dihedral quandle whose order is an odd prime $p$ and $\tau(f)=6$ for a 3-cocycle $f$ of the tetrahedral quandle given in [3. E. Hatakenaka's result given in [7] implies that $\tau\left(\theta_{5}\right) \geq 6$. Using these results, S. Satoh and A. Shima [16, 17] proved that the triple point numbers of 2 - and 3-twist spun trefoil knots are 4 and 6 , respectively.

If the $w$-index of $F$ is zero, then the triple point number of $F$ is also zero, so we have a similar question as in the above paragraph, i.e., for a given 3-cocycle $f$, how many is the minimal number $\omega(f)$ of the $w$-indices of surface links whose quandle cocycle invariants associated with $f$ include a non-zero element? Let $T_{2}$ be a 2-twist spun trefoil. It is known that $w\left(T_{2}\right)=6$ (cf. [6]) and $\Phi_{\theta_{3}}\left(T_{2}\right)=$ $\{0,0,0,2,2,2,2,2,2\}$ (cf. [2]), so $\omega\left(\theta_{3}\right) \leq 6$.

Theorem 1.1. 1 Let $Q$ be a quandle such that for any $x, y \in Q, x=y$ if $x * y=x$. (For example, $Q$ is a dihedral quandle whose order is odd.) Let $f$ be a 3-cocycle of $Q$.

(i) Then, $\omega(f) \geq 6$. In particular, $\omega\left(\theta_{3}\right)=6$.

(ii) If $p$ is an odd prime integer $\neq 3$, then $\omega\left(\theta_{p}\right) \geq 7$.

Remark 1.2. Let $F$ be an $S^{2}$-link and $Q$ be a quandle as in Theorem 1 . For any 3-cocycle $f$, if $\Phi_{f}(F)$ includes non-zero element, then $F$ is non-ribbon, so $w(F) \geq 6$ (cf. [6]). In Theorem 1 (i), we generalize it for surface links with any genus.

Corollary 1.3. For a non-negative integer $g$, there is a surface knot $F$ such that $w(F)=6$ and the genus of $F$ is $g$.

We review a chart description and prove a key proposition (Proposition 2.3) in $\S 2$. In $\S 3$, we define quandle cocycle invariants of surface links in terms of chart descriptions. In $\S 4$, we consider subgraphs of charts with 2,3 or 4 white vertices. In $\S 5$, we prove Theorem 1 and Corollary 1.3 , In $\S 6$, we prove Lemma 5.4 .

\section{Chart Description}

In this section, we review a chart description and prove a key proposition (Proposition 2.3). The original notation of a chart, which is a graph in a 2-disk, was introduced for an "unclosed" surface braid and we can modify it to present a closed surface braid (cf. $\S 23$ in [12]).

An $m$-chart is a (possibly empty) finite immersed one-valent or six-valent graph $\Gamma$ in a 2 -sphere $U_{0}$ satisfying the following conditions:

(i) Every edge is directed and labeled by an integer in $\{1,2, \cdots, m-1\}$.

(ii) For each vertex of degree six, three consecutive edges are directed inward and the other three are directed outward; these six edges are labeled by $i$ and $i+1$ alternately for some $i$.

\footnotetext{
${ }^{1} \mathrm{~T}$. Nagase and A. Shima announced that there is no surface link whose $w$-index is seven in [14], and they may prove it on the numbering series. It follows that if $p \neq 3$, then $\omega\left(\theta_{p}\right) \geq 8$.
} 


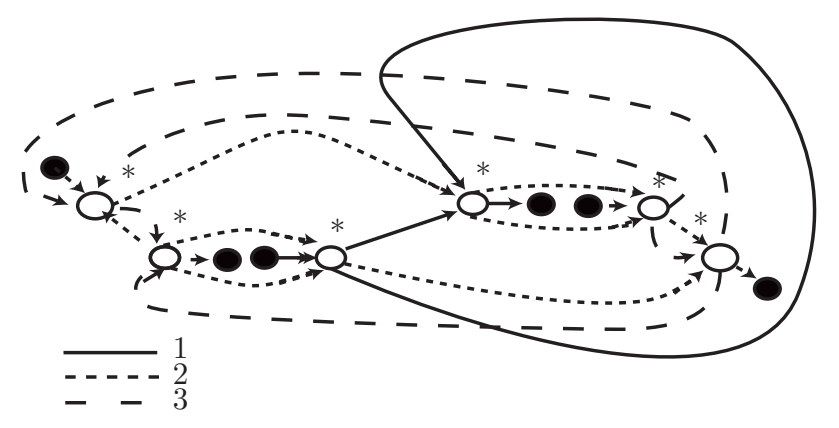

Figure 1

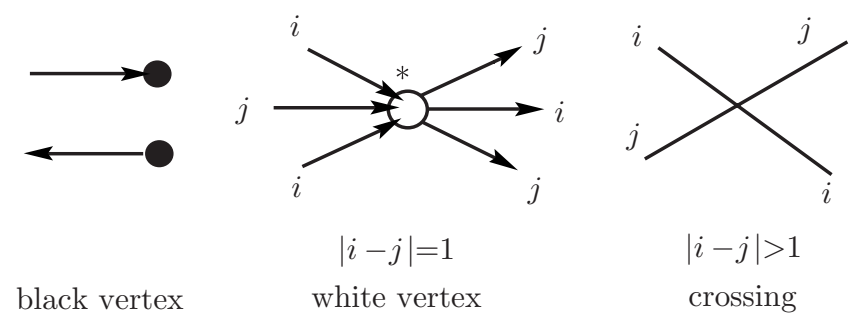

FIGURE 2

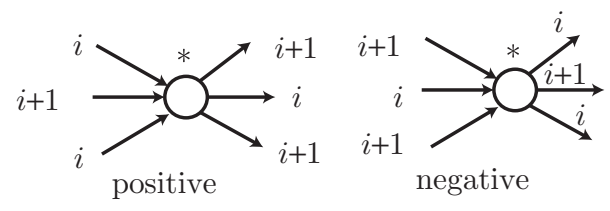

FiguRe 3

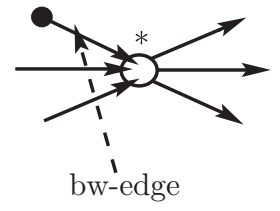

FiguRE 4

(iii) Each singularity of $\Gamma$ is a transverse double point of two edges whose difference in labels is more than one.

An example of a 4-chart is in Fig. 1. We call a one-valent or six-valent vertex a black vertex or white vertex, respectively. We call a singularity a crossing. For each white vertex $W$, we mark ' $*$ ' to the left side region of the left side edge among three consecutive edges directed inward to $W$. See Fig. 2, For a chart $\Gamma$ (or subgraph $G$ of a chart), we denote the number of white vertices of $\Gamma$ (or $G$ ) by $w_{C}(\Gamma)$ (or $\left.w_{C}(G)\right)$. By $c(\Gamma)$ and $b(\Gamma)$, we denote the number of crossings and black vertices of a chart $\Gamma$, respectively. For a white vertex $W$ such that the labels of edges incident to $W$ are $i$ and $i+1, W$ is positive (or negative) if the label of the middle of the three consecutive edges directed inward is $i+1$ (or $i$ ). See Fig. 3. A middle-edge $e$ is an edge that is incident to a white vertex $W$ such that $e$ is the middle of three consecutive edges directed inward to $W$ or the middle of other edges. An edge whose ends are black and white vertices is called a bw-edge. See Fig. 4, A free edge is an edge whose endpoints are black vertices. A closed edge is called a ring if it contains a crossing but not a white vertex nor a black vertex. See Fig. 5 . 


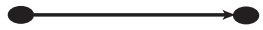

free edge

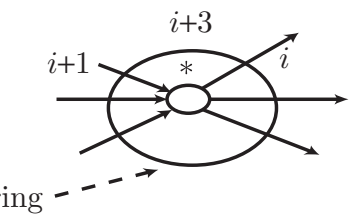

FiguRe 5

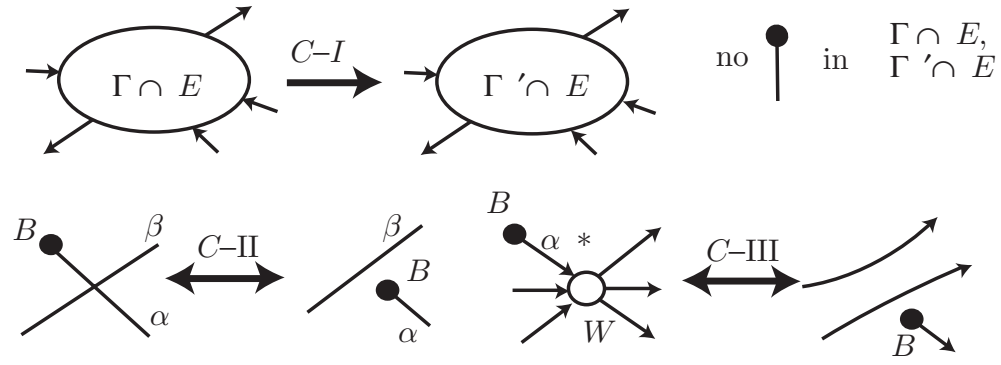

FiguRE 6

The operations listed below (and their inverses) are called a $C_{I^{-}}, C_{I I^{-}}$and $C_{I I I^{-}}$ move, respectively. These moves are called $C$-moves. Two $m$-charts $\Gamma$ and $\Gamma^{\prime}$ are $C$-move equivalent if they are related by a finite sequence of such $C$-moves and ambient isotopies.

$\left(C_{I}\right)$ For a 2-disk $E$ on $U_{0}$ such that $\Gamma \cap E$ and $\Gamma^{\prime} \cap E$ have no black vertices, replace $\Gamma \cap E$ with $\Gamma^{\prime} \cap E$.

$\left(C_{I I}\right)$ Suppose that an edge $\alpha$ is attached to a black vertex $B$ and intersects another edge $\beta$ near $B$. Shorten $\alpha$ to remove the intersection and transmit $B$ across $\beta$.

$\left(C_{I I I}\right)$ Let a black vertex $B$ and a white vertex $W$ be connected by a non-middle edge $\alpha$ of $W$. Remove $\alpha$ and $W$, attach $B$ to the edge of $W$ opposite to $\alpha$, and connect the other four edges in a natural way.

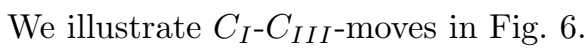

$\mathrm{S}$. Kamada proved that two $m$-charts are $C$-move equivalent if and only if their presented closed surface braids of degree $m$ are equivalent (cf. [11, 12]).

Remark 2.1. In fact, white vertices, black vertices and edges in a chart $\Gamma$ represent triple points, branch points and crossings of one sheet and another one in a diagram of its presented closed surface braid $S$, respectively. The labels and orientations of edges indicate which two sheets of $S$ cross each other and which sheet of them is above another one in $\mathbf{R}^{4}$, respectively. Moreover, the $w$-index of $S$, which is denoted by $w_{B}(S)$, can be redefined in terms of charts as follows:

$$
w_{B}(S)=\min \left\{w_{C}\left(\Gamma^{\prime}\right) \mid \Gamma^{\prime} \text { is } C \text {-move equivalent to } \Gamma\right\} .
$$

Futhermore,

$$
w(F)=\min \left\{w_{B}(S) \mid S \text { is a closed surface braid representing } F\right\} .
$$




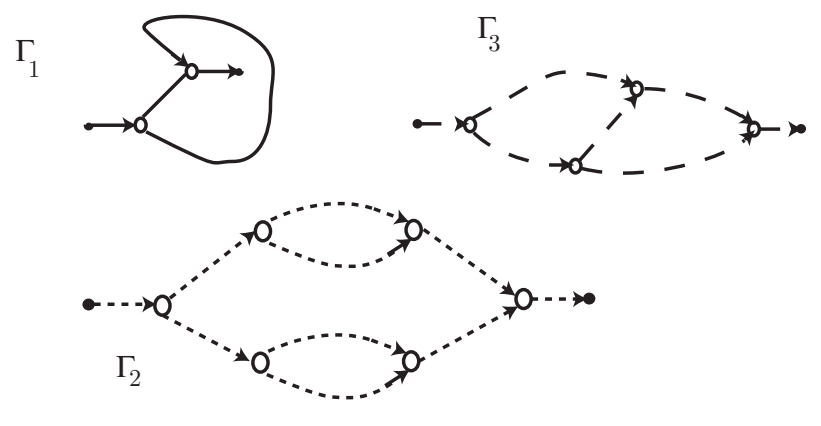

Figure 7

We can also define the $w$-index $\bar{w}_{B}(S)$ of "unclosed" surface braids in a manner similar to $w_{B}(S)$. The original definition of the $w$-index of $F$ was given as the minimal number of $\bar{w}_{B}(S)$ such that $S$ is an "unclosed" surface braid representing $F$. See 9 .

An $m$-chart $\Gamma$ is ribbon if $\Gamma$ is $C$-move equivalent to an $m$-chart $\Gamma^{\prime}$ such that $w_{C}\left(\Gamma^{\prime}\right)=0$. An $m$-chart $\Gamma$ is $C_{23}$-minimal if there is no an $m$-chart $\Gamma^{\prime}$ obtained from $\Gamma$ by at most one $C_{I I}$-move or one $C_{I I I}$-move such that $w_{C}\left(\Gamma^{\prime}\right)+c\left(\Gamma^{\prime}\right)<$ $w_{C}(\Gamma)+c(\Gamma)$.

Lemma $2.2(6])$. For any $m$-chart $\Gamma$, there exists a $C_{23}$-minimal $m$-chart $\Gamma^{\prime}$ such that

- $\Gamma$ is $C$-move equivalent to $\Gamma^{\prime}$, and

- $w_{C}(\Gamma) \geq w_{C}\left(\Gamma^{\prime}\right)$.

Let $\Gamma$ be a $C_{23}$-minimal $m$-chart. For each $i \in\{1, \cdots, m-1\}$, we denote the subgraph consisting of edges with label $i$ and their vertices by $\Gamma_{i}$. For example, for a 4-chart $\Gamma$ in Fig. 1, subcharts $\Gamma_{1}, \Gamma_{2}, \Gamma_{3}$ are illustrated in Fig. 7. Let $X(\Gamma)$ be

$$
X(\Gamma)=\left\{G \subset \Gamma \mid G \text { is a connected component of } \Gamma_{i}, w_{C}(G) \neq 0\right\} .
$$

Proposition 2.3. Let $\Gamma$ be a $C_{23}$-minimal chart. If $|X(\Gamma)| \leq 2$, then $\Gamma$ is ribbon.

Proof. It is obvious that $\Gamma$ is ribbon when $|X(\Gamma)|=0$ and it has not happened that $|X(\Gamma)|=1$, so we suppose that $|X(\Gamma)|=2$. In particular, it is sufficient to consider when $X(\Gamma)=\left\{G, G^{\prime}\right\}$ such that $G \subset \Gamma_{i}$ and $G^{\prime} \subset \Gamma_{i+1}$. Repeating $C_{I}$-moves as in Fig. 8, we deform $\Gamma$ to the union of two disjoint charts $\Gamma^{\prime}$ and $E$ such that all free edges are in $E$ and $G, G^{\prime}$ are in $\Gamma^{\prime}$. We will prove that if $\Gamma^{\prime}$ is ribbon, it follows that $\Gamma$ is $C$-move equivalent to the union of a chart with no white vertices and $E$ with some surrounded loops, and hence $\Gamma$ is ribbon. Since $G \cup G^{\prime}$ is connected, each hoop $h$ and ring $r$ that does not intersect $G \cup G^{\prime}$ bounds a 2-disk $D$ in $U_{0} \backslash\left(G \cup G^{\prime}\right)$, so $h$ and $r$ are made to vanish by $C_{I}$-moves. See Fig. 9. Thus, we assume that $\Gamma^{\prime}=G \cup G^{\prime} \cup R$ such that $R$ consists of rings that intersect $G \cup G^{\prime}$. If $R$ is empty, by the proof of S. Kamada's theorem (Theorem 11) in [8], $\Gamma^{\prime}$ can be deformed to $\Gamma^{\prime \prime}$ with $w_{C}\left(\Gamma^{\prime \prime}\right)=0$, and hence $\Gamma^{\prime}$ is ribbon. Thus, we will prove that any rings can be removed in the intersection with $G \cup G^{\prime}$ by $C$-moves. We remark that the label of each ring in $R$ is neither $i$ nor $i+1$. We consider a ring $r$ in $R$ whose label is $i-1$. The ring $r$ does not intersect $G$ since the difference of labels of $r$ and $G$ 


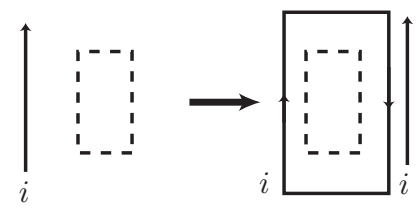

Figure 8

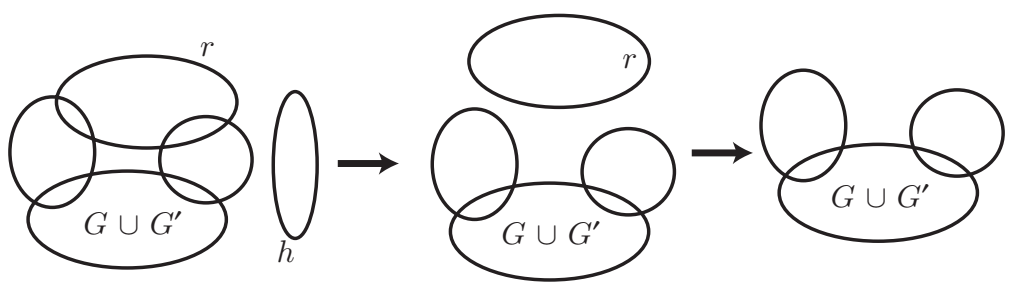

Figure 9

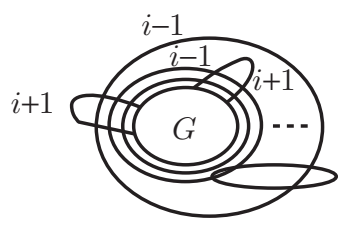

FIGURE 10

is 1 , so $G$ is in the one of two 2-disks given by dividing $U_{0}$ along $r$. We see that all rings with label $i-1$ in $R$ are parallel and surround $G$. See Fig. 10. Let $r^{\prime}$ be an innermost ring with label $j$ among rings in $R$ whose label is less than $i-1$ although $r^{\prime}$ may have an intersection with other rings whose labels are not $j-1, j$ and $j+1$. If $r^{\prime}$ is non-parallel to rings with label $i-1$, then $r^{\prime}$ can be removed since $r^{\prime}$ bounds a 2-disk containing no edges with label $j-1$ or $j+1$. Thus, we suppose that all rings whose labels are less than $i$ are parallel and surround $G$. If $r^{\prime \prime}$ is an outermost ring among rings whose labels are less than $i$, then the intersection of $r^{\prime \prime}$ and $G \cup G^{\prime}$ can be removed by $C$-moves. Repeating this process, the intersections of all rings whose labels are less than $i$ and $G \cup G^{\prime}$ can be removed by $C$-moves. By applying a similar argument to $G^{\prime}$ and rings whose labels are more than $i+1$, the proof of this proposition is complete.

\section{QUANDLE COCYCLE INVARIANTS}

In this section, we will define quandle cocycle invariants in terms of charts. This is a slight modification of the definition given in $\S 11$ of 2 .

3.1. Quandle and quandle cohomology. A quandle is a set $Q$ with a binary operation $*: Q \times Q \longrightarrow Q$ satisfying the following properties:

(i) For any $q \in Q, q * q=q$.

(ii) For any $q_{1}, q_{2} \in Q$, there is a unique $q_{3} \in Q$ such that $q_{1}=q_{3} * q_{2}$. 


$$
C\left(\lambda_{1}\right)=\left(y_{1}, \ldots, y_{m}\right){ }_{\lambda_{1}}^{i} \overbrace{e} \begin{aligned}
& C\left(\lambda_{2}\right)= \\
& \left(y_{1}, \ldots, y_{i-1}, y_{i+1}, y_{i^{*}} y_{i+1}, y_{i+2}, \ldots, y_{m}\right) \\
& \lambda_{2}
\end{aligned}
$$

\section{Figure 11}

(iii) For any $q_{1}, q_{2}, q_{3} \in Q,\left(q_{1} * q_{2}\right) * q_{3}=\left(q_{1} * q_{3}\right) *\left(q_{2} * q_{3}\right)$.

The homology and cohomology for quandles are developed in [2]. For an Abelian group $A$, let $C^{n}(Q ; A)$ be the free Abelian group generated by the maps $f: Q^{n} \longrightarrow$ $A$ such that $f\left(q_{1}, \cdots, q_{n}\right)=0$ if $q_{i}=q_{i+1}$ for some $i \in\{1, \cdots, n\}$. The coboundary map $\delta^{n}: C^{n}(Q ; A) \longrightarrow C^{n+1}(Q ; A)$ is given by

$$
\begin{aligned}
\left(\delta^{n} f\right)\left(q_{1}, \cdots, q_{n+1}\right)= & \sum_{\substack{k=2\\
}}^{n+1}(-1)^{k}\left\{f\left(q_{1}, \cdots, q_{k-1}, q_{k+1}, \cdots, q_{n+1}\right)\right. \\
& \left.f\left(q_{1} * q_{k}, \cdots, q_{k-1} * q_{k}, q_{k+1}, \cdots, q_{n+1}\right)\right\} .
\end{aligned}
$$

The quandle cohomology group $H^{*}(Q ; A)$ is defined by $C^{*}(Q ; A)=\left\{C^{*}(Q ; A), \delta^{*}\right\}$ in a usual manner, and the cocycle group is defined by $Z^{*}(Q ; A)$.

Example 3.1. The set $\{0,1, \cdots, n-1\}$ becomes a quandle under the binary operation $a * b=2 b-a(\bmod n)$, which is called the dihedral quandle of order $n$ and denoted by $R_{n}$. Mochizuki [13] proved that $H^{3}\left(R_{p} ; \mathbf{Z}_{p}\right) \cong \mathbf{Z}_{p}$ for any odd prime $p$. Mochizuki also gave a 3-cocycle

$$
\theta_{p}(x, y, z)=(x-y) \frac{y^{p}+(2 z-y)^{p}-2 z^{p}}{p} \in \mathbf{Z}_{p}
$$

whose cohomology class generates $H^{3}\left(R_{p} ; \mathbf{Z}_{p}\right)$. Here, $y^{p}+(2 z-y)^{p}-2 z^{p}$ is divisible by $p$, so $\theta_{p}$ is well-defined.

3.2. Quandle cocycle invariants of charts. Let $\Gamma$ be an $m$-chart and the set of regions of $D_{2} \backslash \Gamma$ be denoted by $\Sigma(\Gamma)$. A map $C: \Sigma(\Gamma) \longrightarrow Q^{m}$ is a $Q$-coloring of $\Gamma$ if it is such that $C\left(\lambda_{1}\right)=\left(y_{1}, \cdots, y_{m}\right)$ and $C\left(\lambda_{2}\right)=\left(y_{1}, \cdots, y_{i-1}, y_{i+1}, y_{i} *\right.$ $\left.y_{i+1}, y_{i+2}, \cdots, y_{m}\right)$ for each edge $e$ with label $i$ where $\lambda_{1}$ and $\lambda_{2}$ are regions separated by $e$ and $\lambda_{1}$ is on the left side of $e$. See Fig. 11. The set of $Q$-colorings of $\Gamma$ is denoted by $\mathrm{Col}_{Q}(\Gamma)$.

Let $Q$ be a finite quandle and $f \in Z^{*}(Q ; A)$ be a 3 -cocycle of $Q$. Let $C$ be a $Q$-coloring of $\Gamma$. Define the Boltzmann weight at each white vertex $W$ by

$$
W_{f}(W ; C)=\epsilon(W) f\left(y_{i}, y_{i+1}, y_{i+2}\right) \in A,
$$

where $C(\lambda)=\left(y_{1}, \cdots, y_{m}\right), \lambda$ is the region with the asterisk around $W$ and $\epsilon(W)$ is the sign of $W$. We put $W_{f}(C)=\sum W_{f}(W ; C) \in A$. Let

$$
S_{f}(\Gamma)=\left\{W_{f}(C)\right\}_{C \in \operatorname{Col}_{Q}(\Gamma)}
$$

as a multi-set of $A$.

Proposition 3.2. Let $S$ be a closed surface braid that is equivalent to a surface link $F$. Let $\Gamma$ be an $m$-chart presenting $S$. Then, $S_{f}(\Gamma)$ is equal to $\Phi_{f}(F)$.

Proof. It is easy to prove that $S_{f}(\Gamma)$ is equal to $\Phi_{f}(\Gamma)$ in the sense of $\S 11$ of [2]. 
(i) $W$ : positive

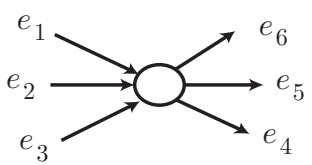

(ii) $W$ : negative

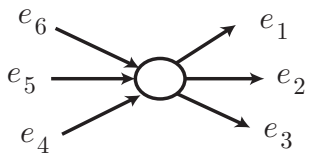

FiguRe 12
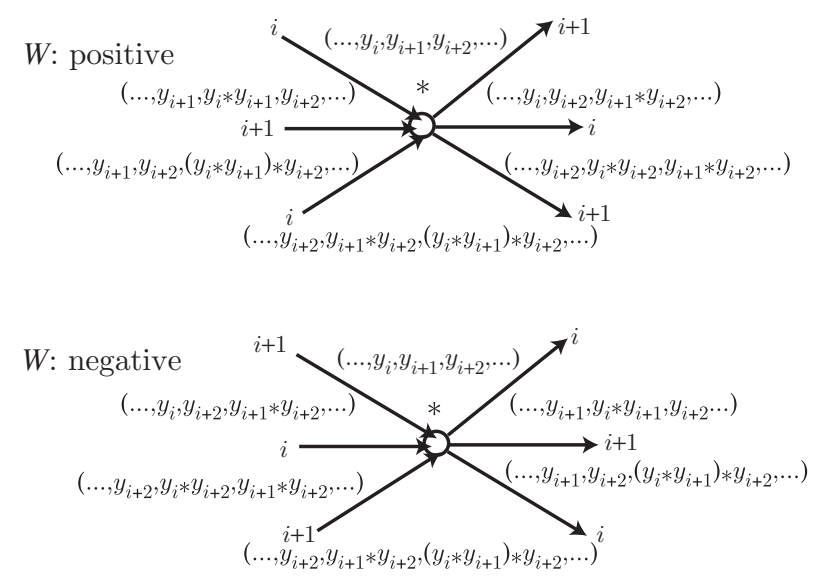

FIGURE 13

3.3. Edge-colorings of a chart. Let $E(\Gamma)$ be the set of edges of an $m$-chart $\Gamma$. A map $E C: E(\Gamma) \longrightarrow Q^{2}$ is a $Q$-edge-coloring of $\Gamma$ if the following conditions are satisfied: (i) if $e$ is an edge connected to a black vertex, then $E C(e)=(a, a)$ for some $a \in Q$, and (ii) if $e_{1}, \cdots, e_{6}$ are edges around a white vertex $W$ as in Fig. 12, then $E C\left(e_{1}\right)=(a, b), E C\left(e_{2}\right)=(a * b, c), E C\left(e_{3}\right)=E C\left(e_{6}\right)=(b, c), E C\left(e_{4}\right)=(a * c, b * c)$ and $E C\left(e_{5}\right)=(a, c)$ for some $a, b, c \in Q$. A $Q$-edge-coloring $E C$ is trivial if the image of $E C$ consists of a unique element $(a, a)$ for some $a \in Q$.

Let $Q$ be a finite quandle and $f \in Z^{*}(Q ; A)$ be a 3 -cocycle of $Q$. Let $E C$ be a $Q$-edge-coloring of $\Gamma$. Then, define the weight at each white vertex $W$ by

$$
\widetilde{W}_{f}(W ; E C)=\epsilon(W) f(a, b, c) \in A,
$$

where $a, b, c$ are given as in the above paragraph and $\epsilon(W)$ is the sign of $W$. Put $\widetilde{W}_{f}(E C)=\sum \widetilde{W}_{f}(E C ; W)$.

Let $C$ be a $Q$-coloring of $\Gamma$. Let $E C$ be a map $E C: E(\Gamma) \longrightarrow Q^{2}$ such that if for each edge $e$ with label $i, \lambda$ is a left-side region of $e$ and $C(\lambda)=\left(y_{1}, \cdots, y_{m}\right)$, then $E C(e)=\left(y_{i}, y_{i+1}\right)$. At each white vertex $W$, it happens that the colors of regions around $W$ by $C$ are as in Fig. 13. Then, we see that $E C\left(e_{1}\right)=\left(y_{i}, y_{i+1}\right), E C\left(e_{2}\right)=$ $\left(y_{i} * y_{i+1}, y_{i+2}\right), E C\left(e_{3}\right)=E C\left(e_{6}\right)=\left(y_{i+1}, y_{i+2}\right), E C\left(e_{4}\right)=\left(y_{i} * y_{i+2}, y_{i+1} * y_{i+2}\right)$ and $E C\left(e_{5}\right)=\left(y_{i}, y_{i+2}\right)$. Thus, $E C$ is a $Q$-edge-coloring. Such a $Q$-edge-coloring is said to be a $Q$-edge-coloring $E C$ induced by $C$.

Lemma 3.3. Let EC be a $Q$-edge-coloring induced by $C$. Then, $W_{f}(C)=\widetilde{W}_{f}(E C)$. 


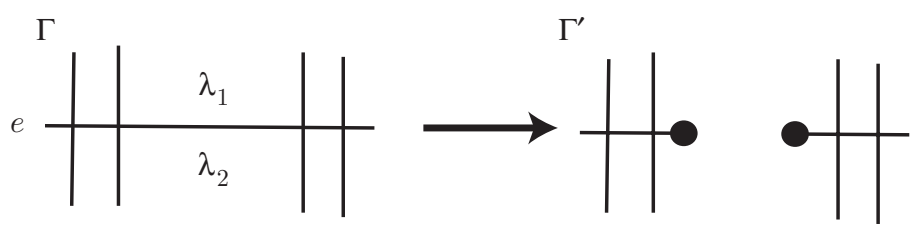

FIGURE 14

Proof. At each white vertex $W, W_{f}(W ; C)=\widetilde{W}_{f}(W ; E C)$. Thus,

$$
W_{f}(C)=\widetilde{W}_{f}(E C) \text {. }
$$

A $Q$-degenerated edge is an edge in $\Gamma$ such that for any $Q$-edge-coloring $E C$ of $\Gamma$, there is an element $q \in Q$ such that $E C(e)=(q, q)$.

Lemma 3.4. Let $\Gamma$ be an $m$-chart with a $Q$-degenerated edge e. Let $\Gamma^{\prime}$ be an $m$ chart given by cutting e and attaching black vertices at its boundary. (See Fig. 14,) Then, $S_{f}\left(\Gamma^{\prime}\right)=S_{f}(\Gamma)$.

Proof. Let $C$ be a $Q$-coloring of $\Gamma$. Then, the $Q$-edge-coloring $E C$ induced by $C$ satisfies that $E C(e)=(q, q)$, so $C\left(\lambda_{1}\right)=C\left(\lambda_{2}\right)$, where $\lambda_{1}, \lambda_{2}$ are opposite regions to each other on $e$. Then, it is easy to see that there is a natural one-to-one correspondence between $\operatorname{Col}_{Q}\left(\Gamma^{\prime}\right)$ and $\operatorname{Col}_{Q}(\Gamma)$ and that corresponding colorings have the same weight at each white vertex. Thus, $S_{f}\left(\Gamma^{\prime}\right)=S_{f}(\Gamma)$. The proof is completed.

\section{Subgraphs of $C_{23}$-Minimal charts}

In this section, we consider any graph $G$ that is an element in $X(\Gamma)$ with $w_{C}(G)=$ $2,3,4$ for a certain $C_{23}$-minimal $m$-chart $\Gamma$ and we always assume that $Q$ is a finite quandle such that $x=y$ if $x * y=x$.

We denote the mirror image and the reverse of an oriented graph (or chart) $G$ in $S^{2}$ by $G^{*}$ and $-G$, respectively. By $-G^{*}$, we mean the reverse of $G^{*}$.

Let $G \in X(\Gamma)$ be a subgraph of a $C_{23}$-minimal chart $\Gamma$. Then, all bw-edges in $\Gamma$ are middle edges. Thus, a white vertex $W$ in $G$ is one of the following four types:

(1) Two edges are directed outward and an edge is directed inward among three edges incident to $W$ and they are not bw-edges.

(2) Two edges are directed outward and an edge is a directed-inward bw-edge among three edges incident to $W$.

(3) An edge is directed outward and two edges are directed inward among three edges incident to $W$ and they are not bw-edges.

(4) An edge is a directed-outward bw-edge and two edges are directed inward among three edges incident to $W$.

See Fig. 15 ,

We denote the number of white vertices with type (1), (2), (3) and (4) by $w_{1}(G), w_{2}(G), w_{3}(G)$ and $w_{4}(G)$, respectively. Since the sum of the edges directed 


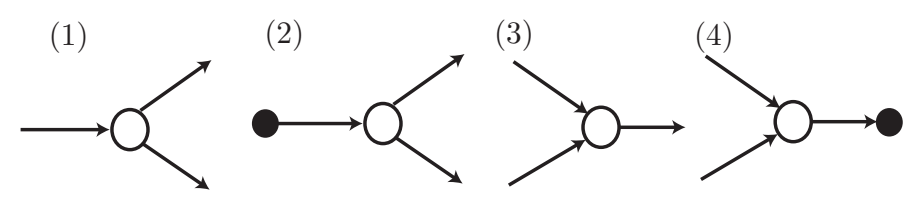

FigURE 15

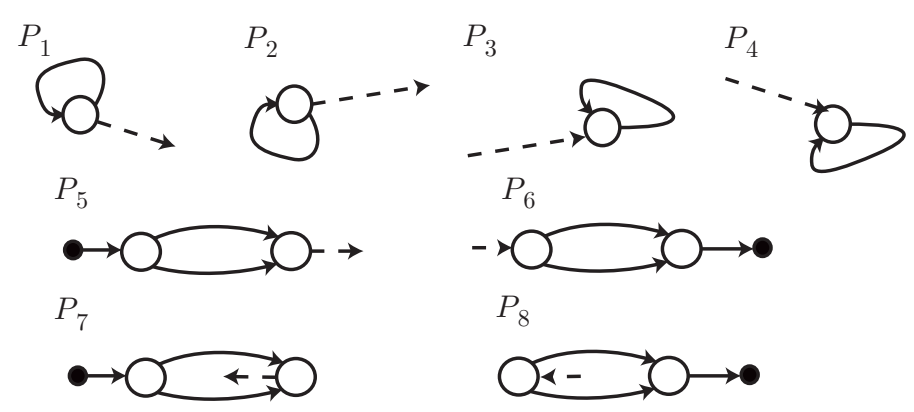

Figure 16

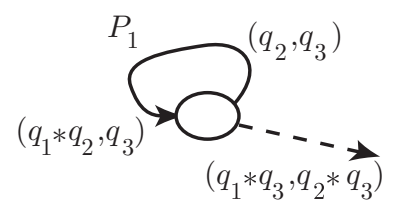

FiguRE 17

inward to white vertices of $G$ is equal to the sum of the edges directed outward to white vertices of $G$, the following equations hold:

$$
\begin{aligned}
2 w_{1}(G)+2 w_{2}(G)+w_{3}(G) & =w_{1}(G)+2 w_{3}(G)+2 w_{4}(G), \\
w_{1}(G)+2 w_{2}(G) & =w_{3}(G)+2 w_{4}(G) .
\end{aligned}
$$

Lemma 4.1. Let $G$ be a graph in $X(\Gamma)$ containing parts $P_{1}, \ldots, P_{8}$ with dotted edge $e$ as illustrated in Fig. 16. Then, $e$ is a Q-degenerated edge.

Proof. In general, the colors of edges around a white vertex $W$ with a sign by a $Q$ edge-coloring are determined locally by three elements in $Q$. This gives a necessary condition for a $Q$-edge-coloring of $\Gamma$ as a simultaneous equation when the signs of all white vertices of $P_{i}$ are fixed. In the case that $W$ is a white vertex in $P_{1}$ whose sign is positive, the simultaneous equation is $\left(q_{2}, q_{3}\right)=\left(q_{1} * q_{2}, q_{3}\right)$. See Fig. 17 Then, $q_{1}=q_{2}$. Since the color of the dotted line $e$ in $P_{1}$ is $\left(q_{1} * q_{3}, q_{2} * q_{3}\right), e$ is a $Q$-degenerated edge. In the other cases, such a simultaneous equation can be given. By solving it, we see that any dotted line in $P_{1}, \ldots, P_{8}$ is a $Q$-degenerated edge.

In Lemmas 4.2, 4.3 and 4.4, we consider the list of subgraphs in $X(\Gamma)$ for a $C_{23}$ $m$-chart $\Gamma$ satisfying the following condition $\star$;

$\star G \in X(\Gamma)$ does not contain $P_{1}, \ldots, P_{4}$ and if $G$ contains parts $P_{5}, \ldots, P_{8}$ with dotted edge $e$, then $e$ is a bw-edge. 


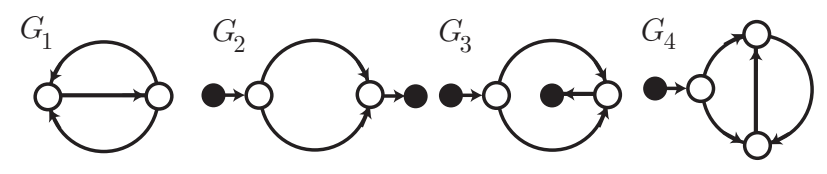

FiguRE 18

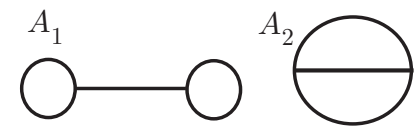

FiguRE 19

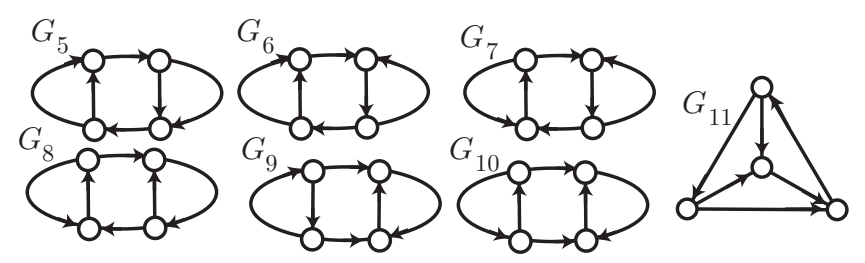

FiguRE 20

Lemma 4.2. Let $G$ with $w(G)=2$ be a graph in $X(\Gamma)$ for a certain $C_{23}$-minimal $m$-chart $\Gamma$. If $G$ satisfies condition $\star$, then $G$ is one of $G_{1}, G_{2}$ and $G_{3}$. Each graph $G_{i}$ is illustrated in Fig. 18.

Proof. By Equation 4.2. we see that $\left(w_{1}(G), w_{2}(G), w_{3}(G), w_{4}(G)\right)=(1,0,1,0)$, $(0,1,0,1)$. First, we consider when $\left(w_{1}(G), w_{2}(G), w_{3}(G), w_{4}(G)\right)=(1,0,1,0)$. By Euler's formula, the number of regions of $S^{2} \backslash N(G)$ is 3, where $N(G)$ is a regular neighborhood of $G$. Thus, $G$ without orientation is one of two graphs $A_{1}, A_{2}$ illustrated in Fig. 19. We see that $A_{1}$ has not satisfied condition $\star$ and $A_{2}$ with orientation is $G_{1}$. See Fig. 18. Second, we consider when $\left(w_{1}(G), w_{2}(G), w_{3}(G), w_{4}(G)\right)=$ $(0,1,0,1)$. By Euler's formula, the number of regions of $S^{2} \backslash N(G)$ is 2 . There are two graphs $G_{2}, G_{3}$ depending on whether two bw-edges are in the same region or not. See Fig. 18

Lemma 4.3. Let $G$ with $w(G)=3$ be a graph in $X(\Gamma)$ for a certain $C_{23}$-minimal $m$-chart $\Gamma$. If $G$ satisfies condition $\star$, then $G$ is one of $G_{4},-G_{4}, G_{4}^{*}$ and $-G_{4}^{*}$. The graph $G_{4}$ is illustrated in Fig. 18.

Proof. By Equation 4.2, we see that $\left(w_{1}(G), w_{2}(G), w_{3}(G), w_{4}(G)\right)=(0,1,2,0)$, $(2,0,0,1)$. By Euler's formula, the number of regions of $S^{2} \backslash N(G)$ is 3 , so $G$ is without one bw-edge $e$ and the orientation is one of the graphs $A_{1}, A_{2}$ illustrated in Fig. 19. We see that $A_{1}$ does not satisfy condition $\star$ wherever $e$ attaches to $A_{1}$. It is a unique choice how to attach $e$ to $A_{2}$, and there are four graphs $G_{4},-G_{4}, G_{4}^{*},-G_{4}^{*}$ depending on the choice of an orientation of $A_{2}$. 


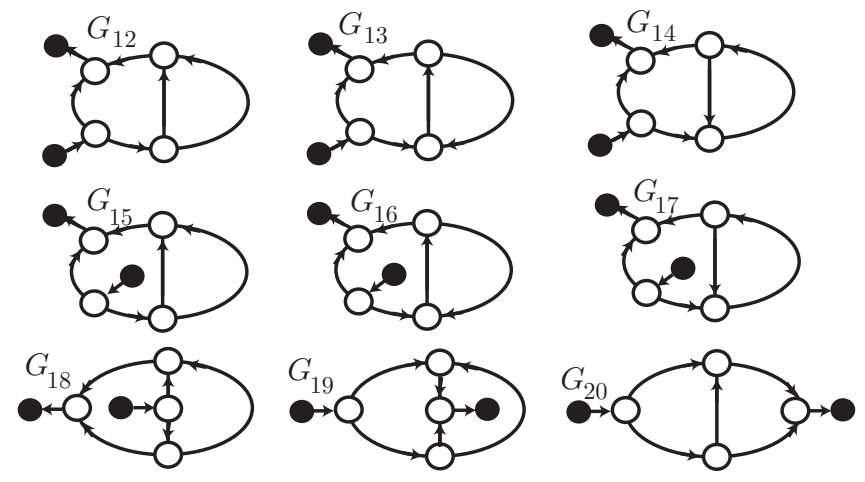

FiguRe 21
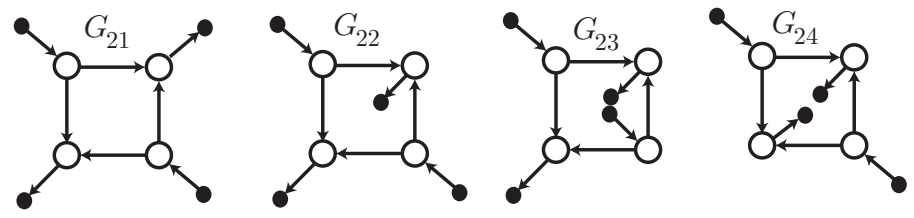

FiguRe 22

Lemma 4.4. Let $G$ with $w(G)=4$ be a graph in $X(\Gamma)$ for a certain $C_{23}$-minimal $m$-chart $\Gamma$. If $G$ satisfies condition $\star$, then $G$ is one of

$$
\begin{aligned}
& G_{5}, \ldots, G_{24}, G_{6}^{*}, G_{7}^{*}, G_{9}^{*}, \ldots, G_{20}^{*}, \\
& \quad-G_{18},-G_{22},-G_{18}^{*} .
\end{aligned}
$$

Each graph $G_{i}$ with $i=5, \ldots, 24$ is illustrated in Figs. 20, 21] and 22.

Proof. By Equation 4.2, we see that

$$
\begin{aligned}
& \left(w_{1}(G), w_{2}(G), w_{3}(G), w_{4}(G)\right) \\
& \quad=(2,0,2,0),(1,1,1,1),(0,2,0,2) .
\end{aligned}
$$

First, we consider when $\left(w_{1}(G), w_{2}(G), w_{3}(G), w_{4}(G)\right)=(2,0,2,0)$. By Euler's formula, the number of regions of $S^{2} \backslash N(G)$ is 4 . Thus, $G$ without orientation is one of the four graphs $A_{3}, A_{4}, A_{5}, A_{6}$ illustrated in Fig. 23. We see that $A_{5}$ and $A_{6}$ do not satisfy condition $\star$. There are ten (or two) graphs $G_{5}, \ldots, G_{10}, G_{6}^{*}, G_{7}^{*}, G_{9}^{*}, G_{10}^{*}$ (or $G_{11}, G_{11}^{*}$ ) depending on the orientation of $A_{3}$ (or $A_{4}$ ). See Fig. 20. Second, we consider when $\left(w_{1}(G), w_{2}(G), w_{3}(G), w_{4}(G)\right)=(1,1,1,1)$. By Euler's formula, the number of regions of $S^{2} \backslash N(G)$ is 3. Thus, $G$ without orientation is one of the two graphs $A_{1}, A_{2}$ illustrated in Fig. 19, If it is $A_{1}$, then $G$ does not satisfy condition $\star$. There are six cases $A_{7}, \ldots, A_{12}$ depending on where we attach two bw-edges to $A_{2}$. See Fig. 24. There are six, three, three, four, two and two graphs depending on the choice of orientation of graphs $A_{7}, \ldots, A_{12}$, respectively. They are graphs $G_{12}, \ldots, G_{20}$ illustrated in Fig. 21, their mirror image and $-G_{18},-G_{18}^{*}$. Third, we consider when $\left(w_{1}(G), w_{2}(G), w_{3}(G), w_{4}(G)\right)=(0,2,0,2)$. By Euler's formula, the number of regions of $S^{2} \backslash N(G)$ is 2 , so $G$ without all bw-edges is a circle. There 


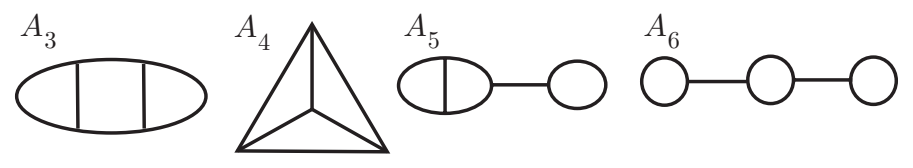

FiguRe 23

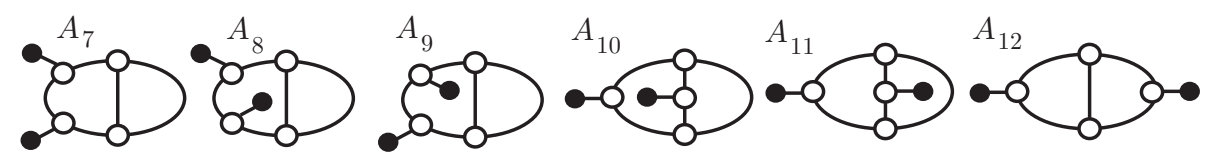

FiguRE 24

are five graphs $G_{21}, \ldots, G_{24},-G_{22}$ depending on where we attach four bw-edges to the circle. This completes the proof.

Remark 4.5. For $G_{1}, \ldots, G_{24}$, we see that

$$
\begin{array}{ll}
G_{i}=G_{i}^{*}=-G_{i}=-G_{i}^{*} & \text { if } i=1,2,3,5,8,21,23,24, \\
G_{i}=-G_{i} \neq G_{i}^{*}=-G_{i}^{*} & \text { if } i=10,11,15,16,17,19,20, \\
G_{i}=G_{i}^{*} \neq-G_{i}=-G_{i}^{*} & \text { if } i=22, \\
G_{i}=-G_{i}^{*} \neq G_{i}^{*}=-G_{i} & \text { if } i=6,7,9,12,13,14, \\
G_{i} \neq-G_{i} \neq G_{i}^{*} \neq-G_{i}^{*} & \text { if } i=4,18 .
\end{array}
$$

\section{Proof of Theorem 1}

In this section, we will prove Theorem 1 and Corollary 1.3

For graphs $G_{1}, \ldots, G_{24}$ given in $\S 4$, let $W_{1}, \ldots, W_{j}$ be white vertices in $G_{i}$ as illustrated in Fig. 25)(i)-(viii), where $W_{C}\left(G_{i}\right)=j$. For a given $C_{23}$-minimal chart $\Gamma$ such that $G_{i} \in X(\Gamma)$ for $i \in\{1, \ldots, 24\}$, let $a_{k}$ be the label of all edges that are incident to $W_{k}$ in $\Gamma \backslash G_{i}$. For $G=-G_{i}, G_{i}^{*},-G_{i}^{*}$, let $W_{1}, \ldots, W_{j}$ and $a_{1}, \ldots, a_{j}$ be white vertices and edges of $G$ corresponding to those of $G_{i}$. We see that each $a_{k}$ is uniquely determined.

Let $\mathfrak{G}_{1}$ and $\mathfrak{G}_{2}$ be the sets of graphs given by

$$
\begin{aligned}
\mathfrak{G}_{1} & =\left\{G_{1}, G_{2}, G_{3}, G_{4}, G_{4}^{*},-G_{4},-G_{4}^{*}\right\}, \\
\mathfrak{G}_{2} & =\left\{G_{5}, \ldots, G_{24}, G_{6}^{*}, G_{7}^{*}, G_{9}^{*}, \ldots, G_{20}^{*},-G_{18},-G_{22},-G_{18}^{*}\right\} .
\end{aligned}
$$

Lemma 5.1. Let $G$ be one of $\mathfrak{G}_{1} \cup \mathfrak{G}_{2}$. Suppose that $\Gamma$ is a $C_{23}$-minimal m-chart such that $G \in X(\Gamma)$.

(a) If $\sum_{k=1}^{j} \widetilde{W}_{f}\left(W_{k} ; E C\right) \neq 0$ for $G \in \mathfrak{G}_{1}$ where $E C$ is a Q-edge-coloring of $\Gamma$, then there are the following cases:

(i) $G=G_{3}$ and $a_{1}=a_{2}$;

(ii) $G=G_{4}, G_{4}^{*},-G_{4},-G_{4}^{*}$ and $a_{1}=a_{2}=a_{3}$.

Furthermore, every middle-edge incident to $G$ in $\Gamma \backslash G$ is not a bw-edge.

(b) If $\sum_{k=1}^{j} \widetilde{W}_{\theta_{p}}\left(W_{k} ; E C\right) \neq 0$ for $G \in \mathfrak{G}_{2}$, where $\theta_{p}$ is Mochizuki's 3-cocycle of the dihedral quandle $R_{p}$ of order $p$ with an odd prime integer $\neq 3$ and $E C$ is an $R_{p}$-edge-coloring of $\Gamma$, then there are the following cases:

(iii) $G=G_{9}, G_{10}, G_{11}, G_{20}, G_{9}^{*}, G_{10}^{*}, G_{11}^{*}, G_{20}^{*}$ and $a_{1}=a_{2}=a_{3}=a_{4}$. 


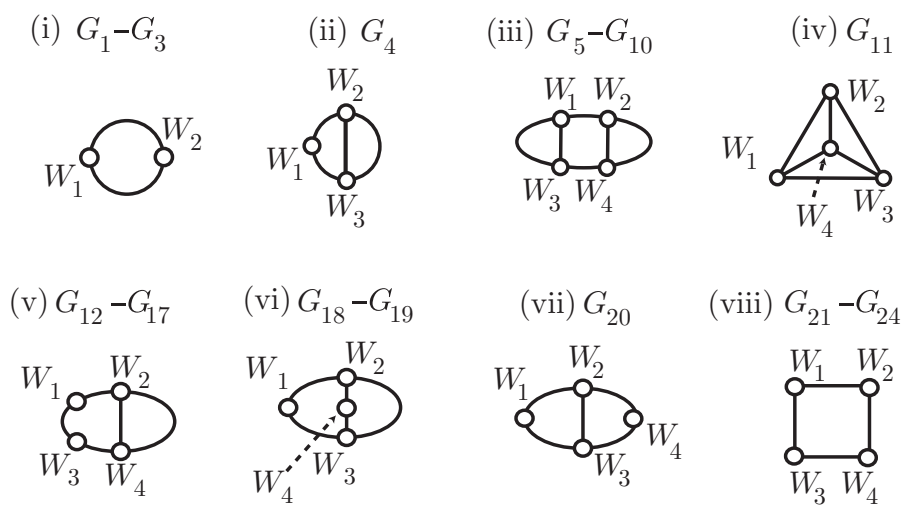

FigURE 25

(iv) $G=G_{18}, G_{19}, G_{22}, G_{24}, G_{18}^{*}, G_{19}^{*},-G_{18},-G_{22},-G_{18}^{*}$ and $a_{1}=a_{2}=a_{3}=$ $a_{4}$.

(v) $G=G_{12}, G_{15}, G_{12}^{*}, G_{15}^{*}$ and $a_{1}=a_{4} \neq a_{2}=a_{3}$.

(vi) $G=G_{15}, G_{15}^{*}$ and $a_{1}=a_{3}, a_{2}=a_{4}$.

(vii) $G=G_{18}, G_{19}, G_{18}^{*}, G_{19}^{*},-G_{18},-G_{18}^{*}$ and $a_{1}=a_{2}=a_{3} \neq a_{4}$.

(viii) $G=G_{19}, G_{19}^{*}$ and $a_{1} \neq a_{2}=a_{3}=a_{4}$.

(ix) $G=G_{22},-G_{22}, G_{24}$ and $a_{1}=a_{2} \neq a_{3}=a_{4}$ or $a_{1}=a_{3} \neq a_{2}=a_{4}$.

(x) $G=G_{23}$ and $a_{1}=a_{2} \neq a_{3}=a_{4}$.

Furthermore, there is a middle-edge incident to $G$ in $\Gamma \backslash G$ that is not a bw-edge when $G=G_{18}, G_{19}, G_{18}^{*}, G_{19}^{*},-G_{18},-G_{18}^{*}$, and every middle-edge incident to $G$ in $\Gamma \backslash G$ is not a bw-edge when $G=G_{12}, G_{15}, G_{19}, G_{20}, G_{22}, G_{23}, G_{24}, G_{12}^{*}, G_{15}^{*}, G_{19}^{*}$, $G_{20}^{*},-G_{22}$.

Proof. We will consider a simultaneous equation as in the proof of Lemma 4.1 for each graph $G \in \mathfrak{G}_{1} \cup \mathfrak{G}_{2}$ when the signs of all white vertices in $G$ are fixed.

When $G=G_{1}$ and the signs of $W_{1}$ and $W_{2}$ are the same (and hence $a_{1} \neq a_{2}$ ), there exist $q_{1}, \ldots, q_{6} \in Q$ such that $\left(q_{1}, q_{3}\right)=\left(q_{4} * q_{5}, q_{6}\right),\left(q_{1}, q_{2}\right)=\left(q_{5}, q_{6}\right)$ and $\left(q_{2}, q_{3}\right)=\left(q_{4} * q_{6}, q_{5} * q_{6}\right)$. See Fig. 26. Solving this simultaneous equation, we have $q_{1}=\ldots=q_{6}$, so the weights of $W_{1}$ and $W_{2}$ are zeros. We see that $\sum_{k=1}^{2} \widetilde{W}_{f}\left(W_{k} ; E C\right)=0$. Similarly, when $G=G_{1}$ and the signs of $W_{1}$ and $W_{2}$ are different (and hence $\left.a_{1}=a_{2}\right)$, we see that $\widetilde{W}_{f}\left(W_{1} ; E C\right)=-\widetilde{W}_{f}\left(W_{2} ; E C\right)$ for any $Q$-edge-coloring $E C$, so $\sum_{k=1}^{2} \widetilde{W}_{f}\left(W_{k} ; E C\right)=0$. Hence, the hypothesis of (a) is not satisfied in this case. Solving similar simultaneous equations given for each $G \in \mathfrak{G}_{1}$ and each choice of signs of white vertices in $G$, we have the cases (i)-(ii).

In the case (i), we see that the weights of $W_{1}$ and $W_{2}$ of $G_{3}$ are $\left(q, q^{\prime}, q\right)$ and $-\left(q^{\prime}, q, q^{\prime}\right)$, respectively. If a middle-edge incident to $G_{3}$ in $\Gamma \backslash G_{3}$ is a bw-edge, then it is required that $q * q^{\prime}=q$ or $q^{\prime} * q=q^{\prime}$ since $\Gamma$ is $C_{23}$-minimal, so $q=q^{\prime}$. Thus, it does not satisfy the hypothesis of (a) in this case. By a similar argument, this completes the proof of (a).

Solving similar simultaneous equations given for $Q=R_{p}$ and $G \in \mathfrak{G}_{2}$ when the signs of all white vertices in $G$ are fixed, we also have cases (iii)-(x). If $G=$ $G_{12}, G_{15}, G_{19}, G_{20}, G_{22}, G_{23}, G_{24}$ and both middle-edges incident to $G$ in $\Gamma \backslash G$ are bw-edges, or if $G=G_{12}, G_{15}, G_{19}, G_{20}, G_{22}, G_{23}, G_{24}, G_{12}^{*}, G_{15}^{*}, G_{19}^{*}, G_{20}^{*},-G_{22}$ and 


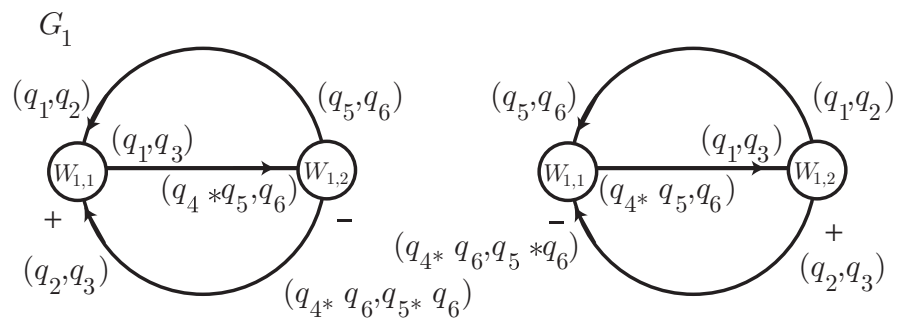

FiguRE 26

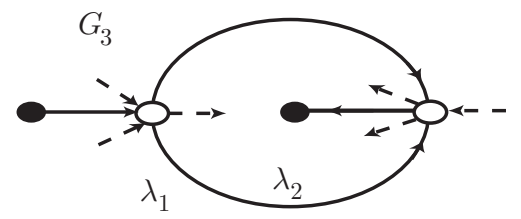

FigURE 27

a middle-edge incident to $G$ in $\Gamma \backslash G$ is a bw-edge, then the hypothesis of (b) is not satisfied. This completes the proof.

Lemma 5.2. In the hypothesis of Lemma $5.1(a), w_{C}(\Gamma) \geq 6$.

Proof. It is sufficient to consider the cases (i) and (ii) in Lemma 5.1 (a). In the case (i), let $\lambda_{1}$ and $\lambda_{2}$ be 2-disks of $S^{2} \backslash N\left(G_{3}\right)$ as in Fig. 27. In $\lambda_{1}$, there are three edges with label $a_{1}$ in $\Gamma$ such that they are not bw-edges and directed inward to white vertices in $G$ by Lemma 5.1 (a). These edges cannot cross $G$, so we need at least two white vertices in $\lambda_{1}$. Similarly, we also need at least two white vertices in $\lambda_{2}$. Thus, $w_{C}(\Gamma) \geq 6$. In the case (ii), by an argument similar to case (i), we also need at least one white vertex in each region of $S^{2} \backslash G$. Thus, $w_{C}(\Gamma) \geq 6$. This completes the proof.

Proof of Theorem 1 $(a)$. There is not a non-ribbon $m$-chart $\Gamma$ with $w_{C}(\Gamma)=1,2,3$. See [6. Thus, we suppose that there exists a $C_{23}$-minimal $m$-chart $\Gamma$ such that $w_{C}(\Gamma)=4,5$ and $S_{f}(\Gamma)$ includes a non-identity element.

Since $S_{f}(\Gamma)$ includes a non-identity element, there are a $Q$-coloring $C$ of $\Gamma$ and a $Q$-edge-coloring $E C$ induced by $C$ such that $\widetilde{W}_{f}(E C)=W_{f}(C) \neq 0$ by Lemma 3.3. By Proposition 2.3, $|X(\Gamma)| \geq 2$. Since $\Gamma$ is a $C_{23}$-minimal $m$-chart, each graph in $X(\Gamma)$ includes at least two white vertices, so $|X(\Gamma)|=2$. Thus, there is a graph $G \in X(\Gamma)$ such that $\sum_{W \in G} \widetilde{W}_{f}(W ; E C) \neq 0$ and $w_{C}(G)=2$ or 3 . If $G$ does not satisfy condition $\star$ in $\S 4$, then $G$ contains one of $P_{1}, \ldots, P_{4}$ or contains one of $P_{5}, \ldots, P_{8}$ whose dotted line is not a bw-edge. Each dotted line in their parts is a $Q$-degenerated edge by Lemma 4.1. Let $\Gamma^{\prime}$ be an $m$-chart given by cutting edges corresponding to such dotted lines in $G$ and attaching black vertices at its boundary. Then, by Lemma 3.4. $S_{f}(\Gamma)=S_{f}\left(\Gamma^{\prime}\right)$. This process divides $G$ into two pieces $H_{1}, H_{2} \in X\left(\Gamma^{\prime}\right)$ such that $W_{C}\left(H_{i}\right)=1$ for some $i$ since $w_{C}(G) \leq 3$. Then, by $C$-moves, $\Gamma^{\prime}$ is $C$-move equivalent to a $C_{23}$-minimal $m$-chart $\Gamma^{\prime \prime}$ such that 


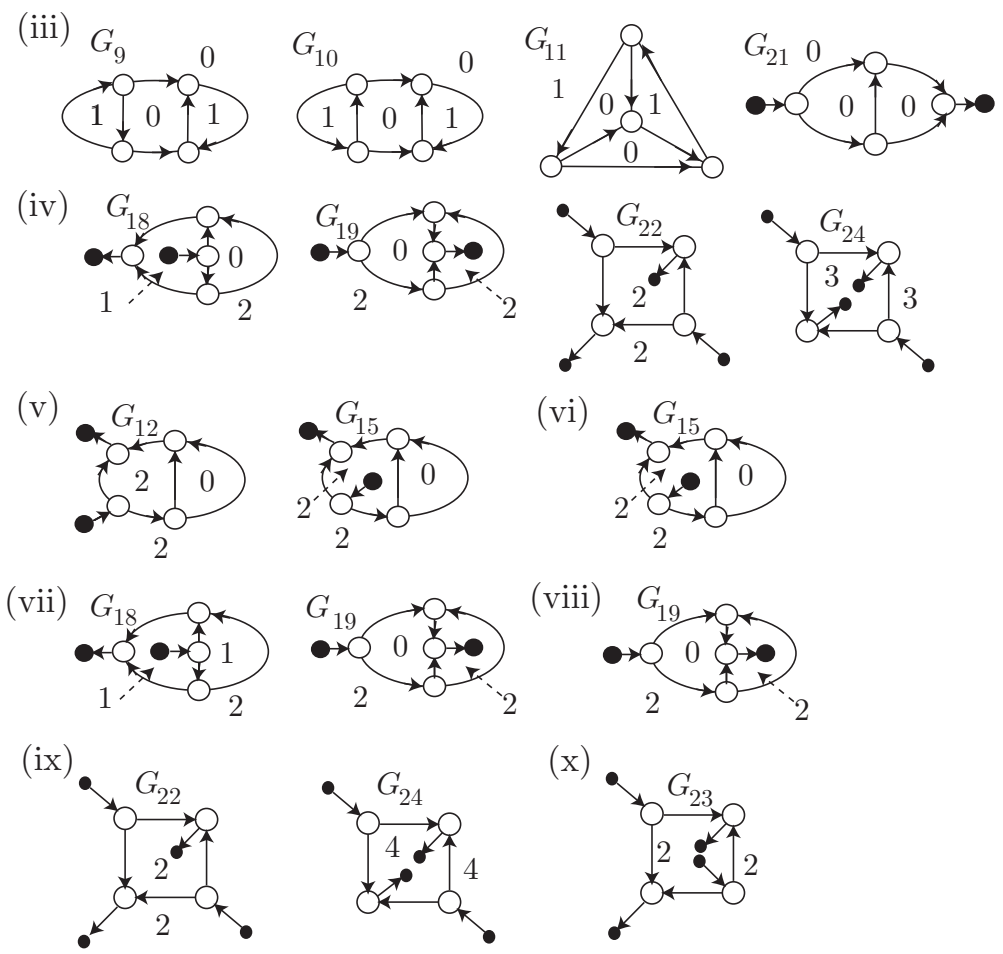

FiguRe 28

$w_{C}\left(\Gamma^{\prime \prime}\right)<w_{C}\left(\Gamma^{\prime}\right)=w_{C}(\Gamma)$. If $w_{C}(\Gamma)=4$, then $\Gamma^{\prime \prime}$ is ribbon, so $S_{f}(\Gamma)=S_{f}\left(\Gamma^{\prime}\right)=$ $S_{f}\left(\Gamma^{\prime \prime}\right)$ consists of zero-elements, so this is a contradiction. If $w_{C}(\Gamma)=5$, then $\Gamma^{\prime \prime}$ is ribbon or $w_{C}\left(\Gamma^{\prime \prime}\right)=4$. There is a contradiction if $\Gamma^{\prime \prime}$ is ribbon. If $w_{C}\left(\Gamma^{\prime \prime}\right)=4$, then we perform the above argument once more after replacing $\Gamma$ with $\Gamma^{\prime \prime}$. If $G$ satisfies condition $\star$, then $G \in \mathfrak{G}_{1}$ by Lemmas 4.2 and 4.3 . Since $\sum_{W \in G} \widetilde{W}_{f}(E C ; W) \neq 0$, $w_{C}(\Gamma) \geq 6$ by Lemma 5.2. This is a contradiction. This completes the proof.

Lemma 5.3. In the hypothesis of Lemma $5.1(b), w_{C}(\Gamma) \geq 7$ when $G$ is satisfied by one of the cases $(i v)-(x)$ of Lemma $5.1(b)$.

Proof. By a similar argument as in the proof of Lemma 5.2, in the cases (iv)-(x) and $G=G_{i}$ for some $i$, we need some white vertices in each region of $S^{2} \backslash N(G)$ as in Fig. 28] so $w_{C}(\Gamma) \geq 7$. Since $G,-G, G^{*},-G^{*}$ require the same number of white vertices in each corresponding region, $w_{C}(\Gamma) \geq 7$ in the other case.

Proof of Theorem 1 (b). By Theorem 1 (a), $\omega\left(\theta_{p}\right) \geq 6$. Thus, suppose that there exists a $C_{23}$-minimal $m$-chart $\Gamma$ such that $w_{C}(\Gamma)=6$ and $S_{\theta_{p}}(\Gamma)$ includes a nonidentity element.

By a similar argument as in the proof of Theorem 10 (a), there exist an $R_{p^{-}}$ coloring $C$ of $\Gamma$ and an $R_{p}$-edge-coloring $E C$ induced by $C$ such that $\widetilde{W}_{\theta_{p}}(E C)=$ $W_{\theta_{p}}(C) \neq 0$, and there is a graph $G$ in $X(\Gamma)$ such that $w_{C}(G)=2,3$ or 4 and $\sum_{W \in G} \widetilde{W}_{\theta_{p}}(E C ; W)$ is non-zero. Moreover, if $G$ does not satisfy condition $\star$ in $\S 4$, there is an $m$-chart $\Gamma^{\prime}$ such that $S_{f}(\Gamma)=S_{f}\left(\Gamma^{\prime}\right)$ and $G$ is divided into two pieces 


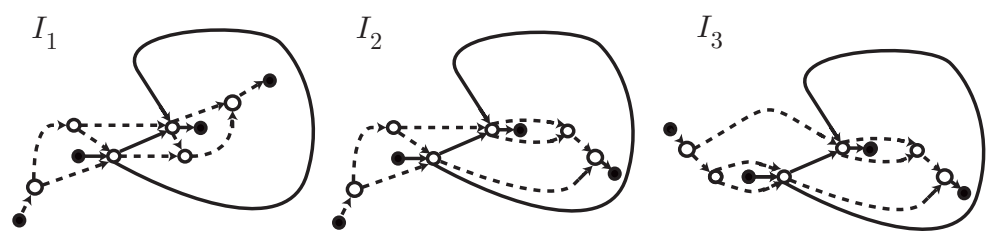

FiguRE 29

$H_{1}, H_{2} \in X\left(\Gamma^{\prime}\right)$. If $w_{C}(G)=2,3$ or $w_{C}(G)=4, w_{C}\left(H_{i}\right)=1$ for some $i$, then $\Gamma$ is $C$ move equivalent to a $C_{23}$-minimal chart $\Gamma^{\prime \prime}$ such that $w_{C}\left(\Gamma^{\prime \prime}\right)<w_{C}\left(\Gamma^{\prime}\right)=w_{C}(\Gamma)=$ 6 and $S_{f}(\Gamma)=S_{f}\left(\Gamma^{\prime}\right)=S_{f}\left(\Gamma^{\prime \prime}\right)$. Thus, $\omega\left(\theta_{p}\right) \leq 5$, so this is a contradiction. If $w_{C}(G)=4$ and $w_{C}\left(H_{1}\right)=w_{C}\left(H_{2}\right)=2$, then we perform the above argument once more after replacing $\Gamma$ with $\Gamma^{\prime \prime}$. If $G$ satisfies condition $\star$, then $G \in \mathfrak{G}_{1} \cup \mathfrak{G}_{2}$ by Lemmas 4.2, 4.3 and 4.4. By Lemmas 5.1 and 5.3, $G$ is satisfied by one of cases (i)-(iii) of Lemma 5.1 .

In the case (i), by the proof of Lemma [5.2, each region of $S^{2} \backslash N\left(G_{3}\right)$ includes exactly two white vertices. There are eight cases $I_{1}, I_{2}, I_{3}, I_{1}^{*}, I_{2}^{*}, I_{3}^{*},-I_{2},-I_{2}^{*}$ such that these white vertices and $G$ are connected by edges with label $a_{1}$ as in Fig. 29. in the other cases, we need other white vertices. Therefore, $\Gamma$ is $C$-move equivalent to one of a $C_{23}$-minimal chart $\Gamma_{1}, \ldots, \Gamma_{5}, \Gamma_{1}^{*}, \ldots, \Gamma_{5}^{*},-\Gamma_{3},-\Gamma_{3}^{*}$ with some loops, rings and free edges. See Fig. 30. Similarly, in cases (ii) and (iii) except for $G=G_{9}, G_{9}^{*}, G_{20}, G_{20}^{*}, \Gamma$ is $C$-move equivalent to a $C_{23}$-minimal chart $\Gamma_{1}, \Gamma_{i},-\Gamma_{i}, \Gamma_{i}^{*},-\Gamma_{i}^{*}$ for $i=6, \ldots, 9$ with some loops, rings and free edges.

If $G=G_{9}$ and $a_{1}=a_{2}=a_{3}=a_{4}$, then it is required that some edges incident to $G$ with label $i$ be connected as in Fig. 33 . Let $J$ be the graph as in Fig. 33. Then, all $R_{p}$-edge-colorings $E C$ of $\Gamma$ are such that $\sum_{W \in J} \widetilde{W}_{\theta_{p}}(E C ; W)=0$ by solving the similar simultaneous equations of $J$ as in the proof of Lemma 4.1. Similarly, if $G=G_{9}^{*}$ and $a_{1}=a_{2}=a_{3}=a_{4}$, then all $R_{p}$-edge-colorings $E C$ of $\Gamma$ are such that $\sum_{W \in J} \widetilde{W}_{\theta_{p}}(E C ; W)=0$.

When $G=G_{20}$ and $a_{1}=a_{2}=a_{3}=a_{4}$, let $e_{1}, \ldots, e_{6}$ be edges incident to $G_{20}$ as in Fig. 34. Similarly, when $G=G_{20}^{*}$ and $a_{1}=a_{2}=a_{3}=a_{4}$, let $e_{1}, \ldots, e_{6}$ be the corresponding edges. If $e_{1}=e_{4}, e_{5}$ or $e_{6}$, then all $R_{p}$-edge-colorings $E C$ of $\Gamma$ are such that $\sum_{W \in G_{20}} \widetilde{W}_{\theta_{p}}(E C ; W)=0$ by solving the similar simultaneous equations as in the proof of Lemma 4.1 Thus, in the case $G=G_{20}, G_{20}^{*}, \Gamma$ is $C$-move equivalent to a $C_{23}$-minimal chart $\Gamma_{4}, \Gamma_{4}^{*}$ with some loops, rings and free edges.

Lemma 5.4. Let $p$ be an odd prime integer $\neq 3$. Then, $\widetilde{W}_{\theta_{p}}(E C)=0$ for an $R_{p}$-edge-coloring $E C$ of $\Gamma_{i}, \Gamma_{i}^{*},-\Gamma_{i}$ and $-\Gamma_{i}^{*}$ with $i=1, \ldots, 9$.

Lemma 5.4 will be proved in $\S 6$.

By Lemma 5.4, for an $R_{p}$-edge-coloring $E C$ of a $C_{23}$-minimal chart $\Gamma$ that is $\Gamma_{i}$ with some loops, rings and free edges, $\widetilde{W}_{\theta_{p}}(E C)=0$. By Lemma 3.3, $S_{\theta_{p}}(\Gamma)$ consists of zero elements. This is a contradiction. This completes the proof of Theorem 1 (b).

Proof of Corollary 1.3, A 4-chart $\Gamma$ illustrated in Fig. 1 presents a 2-twist spun trefoil $T_{2}$. Let $\Gamma^{\prime}$ be a 5-chart illustrated in Fig. 35 and $F$ be a surface knot 

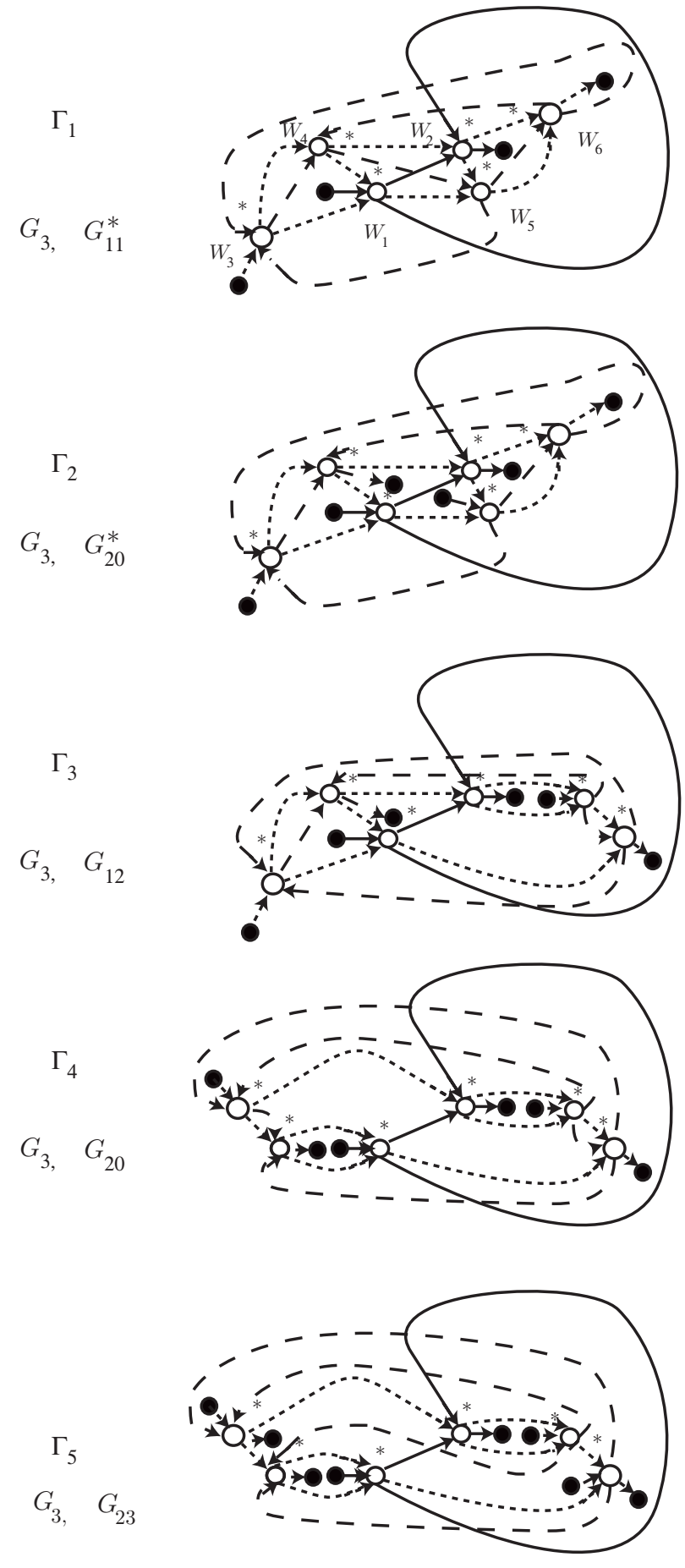

Figure 30 

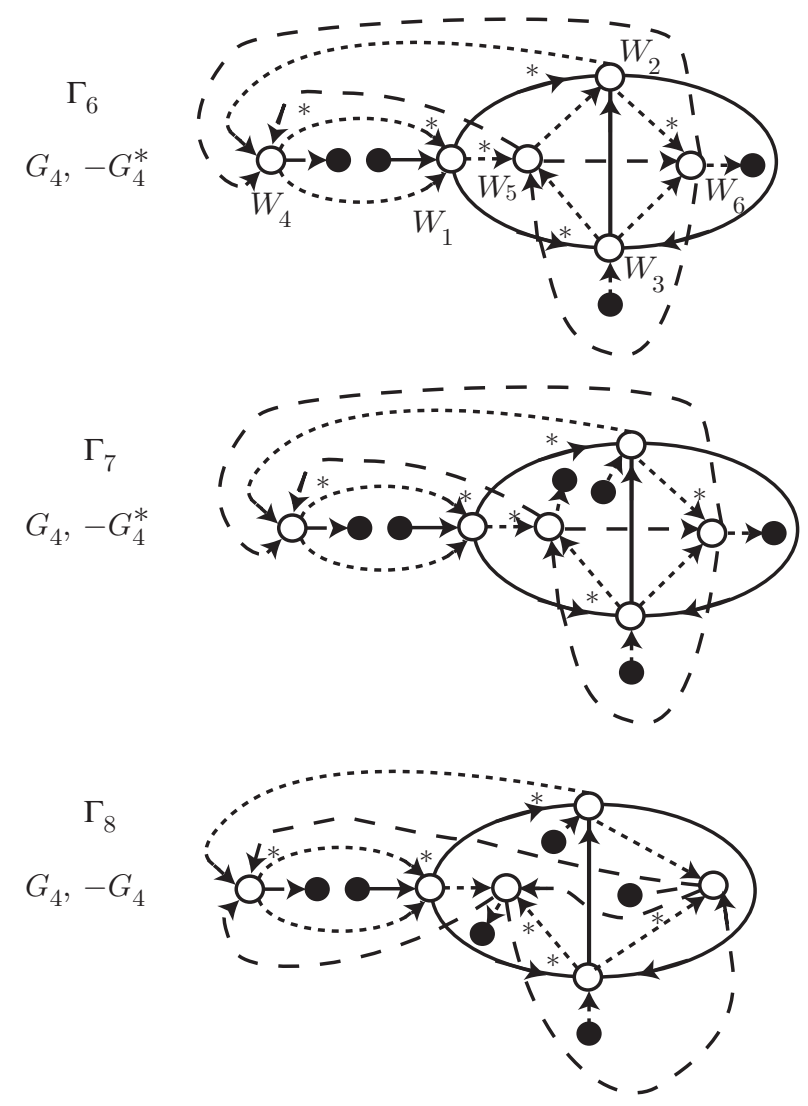

FIGURE 31

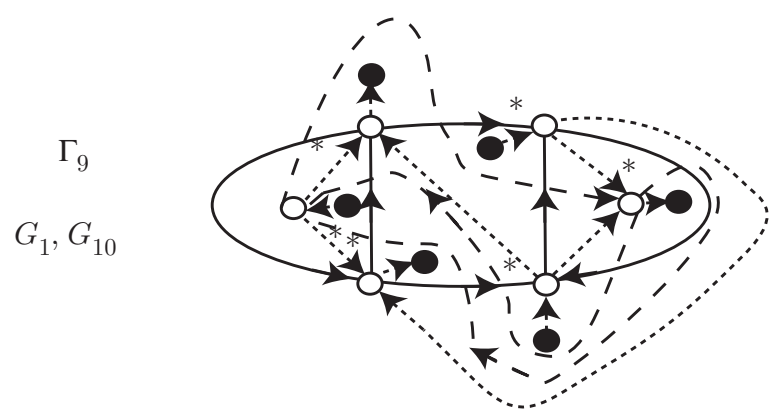

FIGURE 32

presented by $\Gamma^{\prime}$. Then, $S_{\theta_{3}}(\Gamma)=S_{\theta_{3}}\left(\Gamma^{\prime}\right)=\{0,0,0,2,2,2,2,2,2\}$. On the other hand, $F$ is $T_{2}$ with trivial $g_{1} 1$-handles. This completes the proof. 


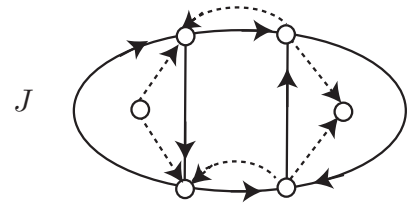

FiguRE 33

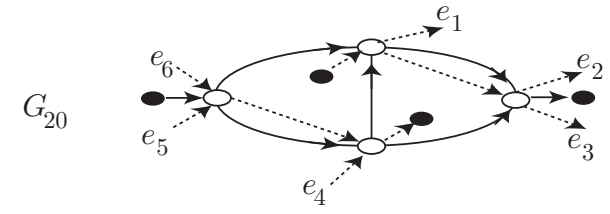

Figure 34

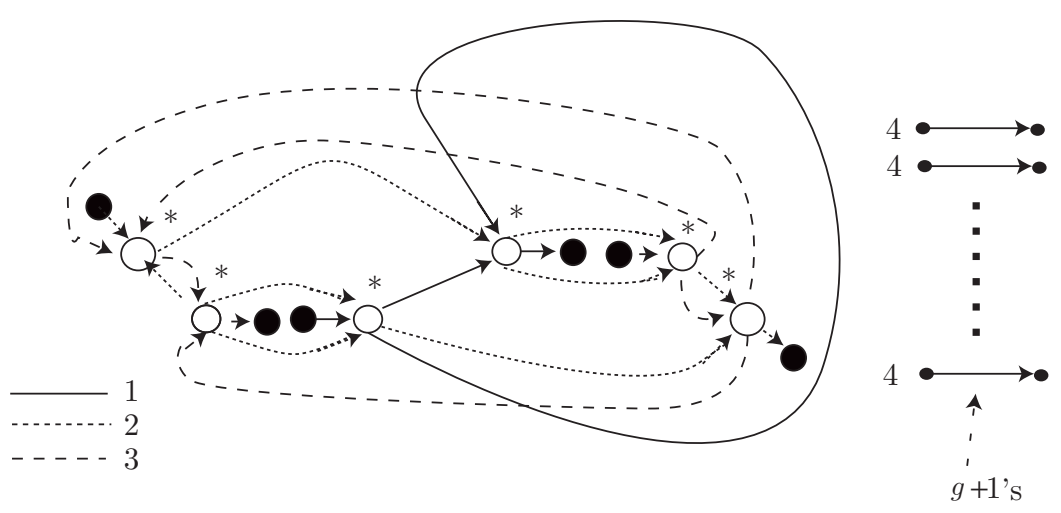

FigURE 35

\begin{tabular}{|c|c|c|}
\hline type & & type (B) \\
\hline & $i$ & $i+2$ \\
\hline$\ldots \ldots$ & $i+1$ & $i+1$ \\
\hline & $i+2$ & \\
\hline
\end{tabular}

FiguRE 36

\section{Proof of Lemma 5.4}

In this section, we will prove Lemma 5.4 and we assume that $p$ is an odd prime integer $\neq 3$.

It is remarked that $\Gamma_{i}=-\Gamma_{i} \neq \Gamma_{i}^{*}=-\Gamma_{i}^{*}$ for $i=1,2,4,5$ and $\Gamma_{i}, \Gamma_{i}^{*},-\Gamma_{i}$ and $-\Gamma_{i}^{*}$ are mutually different for $i=3,6,7,8,9$. In $\S 5$, there are two types (A),(B) of a choice of labels of $\Gamma_{i}$ (cf. Fig. 36), but their presented surface braids $S, S^{\prime}$ are equivalent as surface links. Thus, $S_{i}=S_{i}^{*} \neq-S_{i}=-S_{i}^{*}$ for $i=8$ and $S_{i}=-S_{i}^{*} \neq S_{i}^{*}=-S_{i}$ for $i=6,7$. In particular, we consider only $\Gamma_{i}, \Gamma_{i}^{*}$ for $1=1,6$.

Let $W_{1}, \ldots, W_{6}$ be white vertices of $\Gamma_{1}, \Gamma_{6}$ as in Figs. 30, 31, Let $W_{s}$ be a white vertex of $\Gamma_{i}^{*}$ corresponding to $W_{s}$ of $\Gamma_{i}$ for each $i, s$. By solving the similar simultaneous equations as in the proof of Lemma 4.1, there are $p^{2} R_{p}$-edge-colorings of $\Gamma_{1}, \Gamma_{6}, \Gamma_{1}^{*}, \Gamma_{6}^{*}$ and all $R_{p}$-edge-colorings of $\Gamma_{i}, \Gamma_{i}^{*}$ are trivial for $i \neq 1,6$. Each weight at $W_{s}$ of $\Gamma_{1}, \Gamma_{6}, \Gamma_{1}^{*}, \Gamma_{6}^{*}$ is given in Table 1 . Their colorings are determined by $a, b \in R_{p}$, so we denote them by $E C(a, b)$. 
TABLE 1. $a, b \in R_{p}\left(R_{3}\right), c=a * b, d=(b * a) * b, e=b * a$

\begin{tabular}{|c||c|c|c|c|c|c|}
\hline & $W_{1}$ & $W_{2}$ & $W_{3}$ & $W_{4}$ & $W_{5}$ & $W_{6}$ \\
\hline$\Gamma_{1}$ & $-\theta_{p}(a, b, a)$ & $\theta_{p}(b, a, b)$ & $\begin{array}{c}-\theta_{p}(b * a, \\
a, b * a)\end{array}$ & $\begin{array}{c}-\theta_{p}(b * a, \\
b, a)\end{array}$ & $\begin{array}{c}\theta_{p}(b, a * b, \\
a)\end{array}$ & $\theta_{p}(a, b, a)$ \\
\hline$\Gamma_{1}^{*}$ & $\begin{array}{c}-\theta_{p}(a * d, \\
b * d, c * d)\end{array}$ & $\theta_{p}(b, c, d)$ & $-\theta_{p}(b, c, d)$ & $-\theta_{p}(a, b, d)$ & $\theta_{p}(a, c, d)$ & $\theta_{p}(a, b, c)$ \\
\hline$\Gamma_{6}$ & $-\theta_{p}(a, b, a)$ & $\theta_{p}(a, b, e)$ & $\theta_{p}(b, a, e)$ & $-\theta_{p}(b, a, e)$ & $\begin{array}{c}\theta_{p}(a * b, a, \\
e)\end{array}$ & $\begin{array}{c}\theta_{p}(a * e, \\
b * e, a * e)\end{array}$ \\
\hline$\Gamma_{6}^{*}$ & $-\theta_{p}(a, b, a)$ & $\theta_{p}(e, b, a)$ & $\theta_{p}(e, a, b)$ & $-\theta_{p}(b, a, e)$ & $\begin{array}{c}\theta_{p}(c * b, \\
a * b, a)\end{array}$ & $\theta_{p}(a, b, a)$ \\
\hline
\end{tabular}

Given $\Gamma_{1}$,

$$
\begin{aligned}
\widetilde{W}_{\theta_{p}}(E C(a, b))= & +\theta_{p}(b, a, b)-\theta_{p}(b * a, a, b * a)-\theta_{p}(b * a, b, a)+\theta_{p}(b, a * b, a) \\
= & \frac{b-a}{p}\left(a^{p}+(2 b-a)^{p}-2 b^{p}\right) \\
& -\frac{a-b}{p}\left(a^{p}+(3 a-2 b)^{p}-2(2 a-b)^{p}\right) \\
- & \frac{2(a-b)}{p}\left(b^{p}+(2 a-b)^{p}-2 a^{p}\right) \\
& +\frac{a-b}{p}\left((2 b-a)^{p}+(3 a-2 b)^{p}-2 a^{p}\right) \\
= & 0 .
\end{aligned}
$$

Given $\Gamma_{1}^{*}$,

$$
\begin{aligned}
\widetilde{W}_{\theta_{p}}(E C(a, b))= & -\theta_{p}(a * d, b * d, c * d)-\theta_{p}(a, b, d)+\theta_{p}(a, c, d)+\theta_{p}(a, b, c) \\
= & -\frac{b-a}{p}\left((2 d-b)^{p}+d^{p}-2(2 d-c)^{p}\right) \\
& -\frac{a-b}{p}\left(b^{p}+(2 d-b)^{p}-2 d^{p}\right) \\
+ & \frac{2(a-b)}{p}\left(c^{p}+(2 d-c)^{p}-2 d^{p}\right)+\frac{a-b}{p}\left(b^{p}+d^{p}-2 c^{p}\right) \\
= & 0 .
\end{aligned}
$$

Given $\Gamma_{6}$,

$$
\begin{aligned}
\widetilde{W}_{\theta_{p}}(E C(a, b))= & -\theta_{p}(a, b, a)+\theta_{p}(a, b, c)+\theta_{p}(a * b, a, c)+\theta_{p}(a * c, b * c, a * c) \\
& =-\frac{a-b}{p}\left(b^{p}+c^{p}-2 a^{p}\right)+\frac{a-b}{p}\left(b^{p}+(2 c-b)^{p}-2 c^{p}\right) \\
& +\frac{2(b-a)}{p}\left(a^{p}+(2 c-a)^{p}-2 c^{p}\right) \\
& \quad+\frac{b-a}{p}\left((2 c-b)^{p}+c^{p}-2(2 c-a)^{p}\right) \\
= & 0 .
\end{aligned}
$$

Given $\Gamma_{6}^{*}$,

$$
\begin{aligned}
\widetilde{W}_{\theta_{p}}(E C(a, b)) & =+\theta_{p}(c, b, a)+\theta_{p}(c, a, b)-\theta_{p}(b, a, c)+\theta_{p}(c * b, a * b, a) \\
& =\frac{2(a-b)}{p}\left(b^{p}+c^{p}-2 a^{p}\right)+\frac{a-b}{p}\left(a^{p}+(2 b-a)^{p}-2 b^{p}\right) \\
& -\frac{b-a}{p}\left(a^{p}+(2 c-a)^{p}-2 c^{p}\right) \\
& \quad+\frac{b-a}{p}\left((2 b-a)^{p}+(2 c-a)^{p}-2 a^{p}\right) \\
& =0 .
\end{aligned}
$$


Since all $R_{p}$-edge-colorings of $\Gamma_{i}, \Gamma_{i}^{*}$ are trivial for $i \neq 1,6$ and the above calculation results are zero, we have Lemma 5.4 .

Remark 6.1. Let $\Gamma$ be a chart for one of $\Gamma_{i}, \Gamma_{i}^{*},-\Gamma_{i},-\Gamma_{i}^{*}$ for $i=5,7,9$. Then, $\Phi_{\theta_{3}}(\Gamma)$ includes non-zero elements.

\section{REFERENCES}

1. S. Asami and S. Satoh, An infinite family of non-invertible surfaces in 4-space, Bull. London Math. Soc. 37 (2005), 285-296. MR2119028 (2005k:57044)

2. J. S. Carter, D. Jelsovsky, S. Kamada, L. Langford and M. Saito, Quandle cohomology and state-sum invariants of knotted curves and surfaces, Trans. Amer. Math. Soc., 355 (2003), 3947-3989. MR.1990571 (2005b:57048)

3. J. S. Carter, D. Jelsovsky, S. Kamada and M. Saito, Computations of quandle cocycle invariants of knotted curves and surfaces, Adv. in Math., 157 (2001), 36-94. MR1808844 (2001m:57009)

4. J. S. Carter, M. Saito and S. Satoh, Ribbon concordance of surface-knots via quandle cocycle invariants, J. Aust. Math. Soc. 80 (2006), 131-147. MR2212320 (2006k:57066)

5. R. Fenn, C. Rourke and B. Sanderson, James bundles and applications, Proc. London Math. Soc. (3) 89 (2004), 217-240. MR2063665 (2005d:55006)

6. I. Hasegawa, The lower bound of the w-indices of non-ribbon surface-links, Osaka J. Math., 41 (2004), 891-909. MR2116344 (2005k:57045)

7. E. Hatakenaka, An estimate of the triple point numbers of surface-knots by quandle cocycle invariants, Topology Appl. 139 (2004), 129-144. MR.2051101(2005d:57036)

8. S. Kamada, Surfaces in $R^{4}$ of braid index three are ribbon, J. Knot Theory Ramifications, 1 (1992), 137-160. MR 1164113 (93h:57039)

9. S. Kamada, 2-dimensional braids and chart descriptions, Topics in Knot Theory, 277287, NATO ASI series C, 399 (Erzurum/Turkey 1992), Kluwer Academic Publishers, 1992. MR 1257915

10. S. Kamada, A characterization of groups of closed orientable surfaces in 4-space, Topology, 33 (1994), 113-122. MR.1259518 (95a:57002)

11. S. Kamada, An observation of surface braids via chart description, J. Knot Theory Ramifications, 4 (1996), 517-529. MR.1406718 (97j:57009)

12. S. Kamada, Braid and knot theory in dimension four, Math. Surveys Monogr. 95, Amer. Math. Soc., 2002. MR.1900979(2003d:57050)

13. T. Mochizuki, Some calculations of cohomology groups of finite Alexander quandles, J. Pure Appl. Algebra 179 (2003), 287-330. MR1960136 (2004b:55013)

14. T. Nagase, A. Shima, Properties of minimal charts and their applications. I, J. Math. Sci. Univ. Tokyo 14 (2007), 69-97. MR2320385 (2008c:57040)

15. M. Ochiai, T. Nagase, A. Shima, There exists no minimal n-chart with five white vertices, Proc. Sch. Sci. Tokai Univ. 40 (2005), 1-18. MR2138333 (2006b:57035)

16. S. Satoh, A. Shima, The 2-twist-spun trefoil has the triple point number four, Trans. Amer. Math. Soc. 356 (2004), 1007-1024. MR.1984465 (2004k:57032)

17. S. Satoh, A. Shima, Triple point numbers and quandle cocycle invariants of knotted surfaces in 4-space, New Zealand J. Math. 34 (2005), 71-79. MR2141479(2006e:57031)

18. O. Ya. Viro, Lecture given at Osaka City University, September, 1990.

Graduate School of Science, Osaka City University, 3-3-138 Sugimoto Sumiyoshi-ku, OSAKA 558-8585, JAPAN

E-mail address: iwakiri@sci.osaka-cu.ac.jp 